\title{
Multiracial variations in anatomical location of the occipital artery and nerve complex: a key to avoiding injury prior to strip harvesting
}

\author{
Theresa Marie R. Cacas, MD, Damkerng Pathomvanich, MD, Kulakarn Amonpattana, MD Bangkok, Thailand \\ theresareyesderm@yahoo.com
}

\section{Introduction}

The occipital artery and nerve complex is an important anatomical structure that must be considered in hair transplantation. Every effort should be made to identify its location since trauma to these vital structures can cause significant patient morbidity. Most hair transplant surgeons often do not pay much attention to the occipital artery and nerve, which can lead to complications such as bleeding, hematoma, the possibility of post-surgical anagen effluvium, hypo-/hyperesthesia, neuromas, and neuralgias. ${ }^{1,2}$

In hair transplantation, both the nerve and artery become susceptible to trauma with donor dissection at the posterior occipital scalp. Chances of transection are even higher if tumescent anaesthesia is not administered appropriately, ${ }^{3,4}$ and if anatomical landmarks for these structures are not considered during the overall planning of donor harvesting.

Current studies available to identify these structures use palpable bony landmarks as reference points. ${ }^{5-8}$ These landmarks, however, vary among patients and multiracial groups, and may even be absent in some individuals. ${ }^{5,7}$ This study aims to show how the reflection of the skin of the external ear and scalp is easily identifiable and is a useful landmark in identification of the occipital artery-nerve complex.

\section{Objectives}

The three objectives of this study were:

1. To measure the distance from the reflection of the skin of the external ear and scalp to the point of maximal occipital artery pulsations on the right and left side.

2. To measure the distance between the points of maximal occipital artery pulsations on the right and left side.

3. To compare the means of the above measurements among males, females, and different racial groups.

\section{Materials and Methods}

This study included a total of 100 patients and female staff volunteers at the DHT clinic in Bangkok, Thailand, from August to November 2009.

The safe donor area was selected using the occipital protuberance as a reference point. All patients were placed in the prone position and the occipital artery pulsations were located on both sides by starting palpation from behind the ears. The fingertips were used to feel for occipital artery pulsations moving toward the midline, and running superiorly and inferiorly, until the maximal point of occipital artery pulsation was felt. This spot was marked using gentian violet with a vertical line in the sagittal plane. A horizontal line measuring the distance from reflection of the skin of the external ear and scalp, beginning at the uppermost point of the ear and crossing the vertical mark at 90 degrees or perpendicularly, was measured with a ruler in centimeters and recorded (Figure 1). The distances between the right and left pulsations were recorded as well (Figure 2). Identification of occipital artery pulsations, marking with gentian violet, and measurements were done by a single observer. Data was analyzed by taking the mean and range of recorded measurements on the right and on the left, and the distance between pulsations. These recorded means were compared among different sexes and racial groups.

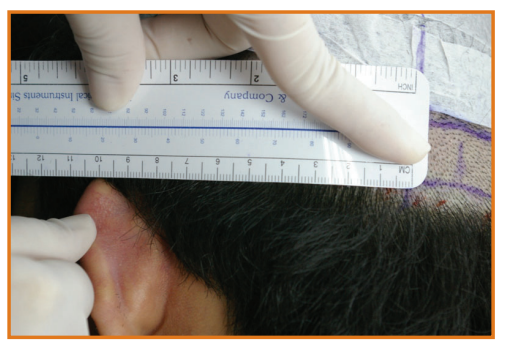

Figure 1. Measuring horizontal distance in centimeters from the superior reflection of skin of the external ear and scalp until the point of maximal occipital artery pulsation as marked with gentian violet.

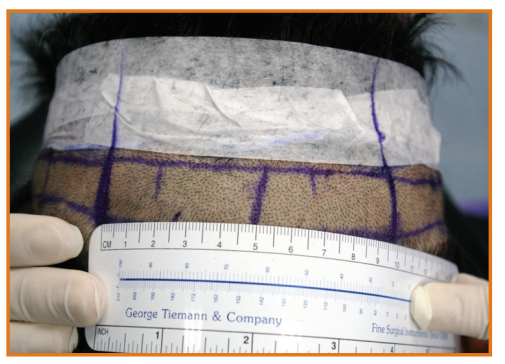

Figure 2. Measuring the distance between occipital artery pulsations.

\section{Results}

Out of the 100 patients included in the study, 86 were male and 14 were female. The mean age was 33.23 with a range of 20-70 years. There were 63 Asian patients, 36 Caucasian patients, and 1 patient of African descent. Of the Asian patients, 46 were Oriental, 6 were Middle Eastern, and 11 were Indian. Symmetrical measurements of the distance between the reflection of the skin of the external ear and the occipital artery pulsations bilaterally were obtained in 55 patients, while 45 had asymmetrical distances (Table 1).

It was observed that in the majority of patients, the maximal point of occipital artery pulsations can be felt at the level of the nuchal ridge. Pulsations can still be felt superior to this point, but they become weaker.

For the entire study population, average measurements of the distance between the reflection of the skin of the external ear and scalp to the occipital artery pulsations was $8.44 \mathrm{~cm}$ (range $6.5-10.5 \mathrm{~cm}$ ) on the right side and $8.42 \mathrm{~cm}$ (range $7-10 \mathrm{~cm}$ ) on the left. The average distance between the right and left occipital artery pulsations was $10.54 \mathrm{~cm}$ (range $8-13.5 \mathrm{~cm}$ ) (Table 2 ). 
Multiracial variations

from page 127

\begin{tabular}{|lr|}
\hline No. of Patients & 100 \\
Sex & \\
$\quad$ Male & 86 \\
$\quad$ Female & 14 \\
Age & \\
Mean & 33.23 years \\
Range & $20-71$ years \\
Race & \\
Asian & 63 \\
Caucasian & 36 \\
African descent & 1 \\
Asian population & 46 \\
Oriental & 6 \\
Middle East & 11 \\
Indian & \\
Measurements between ROA and LOA \\
Symmetrical \\
Asymmetrical & 55 \\
& 45 \\
Table 1. Data of patients Included in the study.
\end{tabular}

\begin{tabular}{|lccc|}
\hline Population & ROA $(\mathbf{c m})$ & LOA $(\mathbf{c m})$ & BET $(\mathbf{c m})$ \\
Total population & 8.44 & 8.42 & 10.54 \\
Asian & & & \\
$\quad$ Oriental & 8.57 & 8.51 & 10.52 \\
$\quad$ Middle East & 8.66 & 8.50 & 10.50 \\
Indian & 7.18 & 7.22 & 10.04 \\
Caucasian & 8.70 & 8.61 & 11.04 \\
African descent & 8.00 & 8.00 & 11.50 \\
Asian Female & 8.39 & 8.64 & 9.67 \\
\end{tabular}

ROA-distance from reflection of skin of the external ear and scalp to maximal point of occipital artery pulsation at the right side

LOA-distance from reflection of skin of the external ear and scalp to maximal point of occipital artery pulsation at the left side

BET-distance between occipital artery pulsations

Table 2. Mean measurements of distance from reflection of skin of the external ear and scalp to right and left maximal occipital artery pulsations, and mean distance between pulsations.

The mean distance of occipital artery measurements was also identified and compared for the different racial groups:

- The Asian Oriental population showed a mean distance of $8.57 \mathrm{~cm}$ for the right side (range $7-10.5 \mathrm{~cm}$ ) and $8.51 \mathrm{~cm}(7-10 \mathrm{~cm})$ for the left; mean distance between pulsations was $10.52 \mathrm{~cm}$ (range $8.5-$ $13.5 \mathrm{~cm})$.

- The Asian Middle East population showed a mean distance of $8.66 \mathrm{~cm}$ for the right (range $8-9 \mathrm{~cm}$ ) and $8.5 \mathrm{~cm}$ (range $7-9.5 \mathrm{~cm}$ ) for the left; mean distance between pulsations was $10.5 \mathrm{~cm}$ (range $9.5-12.5 \mathrm{~cm}$ ).

- The Asian Indian population showed a mean distance of $7.18 \mathrm{~cm}$ for the right (range $7.5-9 \mathrm{~cm}$ ) and $7.22 \mathrm{~cm}$ (range $8-9 \mathrm{~cm}$ ) for the left; mean distance between the artery pulsations was $10.04 \mathrm{~cm}$ (range $9.5-12.5 \mathrm{~cm})$.

- All female patients were Asian Oriental and mean measurements showed an average of $8.39 \mathrm{~cm}$ (range
$7-10.5 \mathrm{~cm}$ ) for the right and 8.64 (range $7-10.5 \mathrm{~cm}$ ) for the left; mean distance between arterial pulsations was $9.67 \mathrm{~cm}$ (range $8-13.5 \mathrm{~cm}$ ).

- The Caucasian population showed a mean distance of $8.70 \mathrm{~cm}$ for the right side (range $6.5-10.5 \mathrm{~cm}$ ) and $8.61 \mathrm{~cm}$ (range $7-10 \mathrm{~cm}$ ) for the left; mean distance between pulsations was $11.04 \mathrm{~cm}$ (range $7.5-14 \mathrm{~cm}$ ).

- The patient of African descent showed a distance of $8 \mathrm{~cm}$ on the right, $8 \mathrm{~cm}$ on the left, and $11.5 \mathrm{~cm}$ between arterial pulsations.

\section{Discussion}

In hair transplantation surgery, one important anatomical structure that has to be considered in donor harvesting and closure is the occipital artery and nerve complex. The course of the occipital artery can be divided into three segments. ${ }^{9}$ The first, or digastric, segment shows that the occipital artery originates from the external carotid opposite the facial artery at the inferior margin of the digastric muscle. It ascends to the interval between the transverse process of the atlas and the mastoid process of the temporal bone, and passes horizontally backward, grooving the surface of the latter bone. At this course, the artery is being covered by the sternocleidomastoid, splenius capitis, longissimus capitis, and digastricus, and is resting upon the rectus capitis lateralis, obliquus superior, and semispinalis capitis. The second, or suboccipital, segment extends from the occipital groove where it then changes its course and runs vertically upward piercing the fascia connecting the cranial attachment of the trapezius with the sternocleidomastoideus to the superior nuchal line. The third, or terminal, segment corresponds to the subgaleal segment just above the superior nuchal line and ascends in a tortuous course in the superficial fascia of the scalp, where it divides into numerous branches. It divides into an ascending branch, a descending branch, and a transverse branch at the nuchal border of the splenius capitis and sternocleidomastoid attachments. ${ }^{10}$ Some branches reach as high as the vertex of the skull and anastomose with the posterior auricular and superficial temporal arteries. This artery supplies blood to the back of the scalp and to the sternomastoid muscles.

The greater occipital nerve has been described as the largest purely sensory nerve in the body. It arises from the dorsal ramus of $\mathrm{C} 2$ deep to the inferior oblique muscle where it branches. The medial branch is the greater occipital nerve, which runs transversely along the inferior oblique and is covered by the splenius capitis, the longissimus, and the semispinalis muscles. The nerve then turns upward to pierce the semispinalis capitis. Here, the nerve runs rostrolaterally before emerging into the scalp by piercing the aponeurotic fibrous attachment of the trapezius and sternocleidomastoid to the superior nuchal line. Immediately below the superior nuchal line, the nerve divides into several terminal branches; medial branches innervate occipital skin and the lateral branches pass into the region behind the pinna."

The point after which the nerve traverses the trapezius muscle aponeurosis to travel within the subcutaneous tissue at the superior nuchal line is the segment of greatest concern during donor harvesting and closure. It is within this area that the occipital nerve is intimately associated to the 
occipital artery and pulsations of the occipital artery can be felt at this point as well..$^{5,12}$ The occipital nerve was shown to always lie superior to the artery and its width increasing in size from a mean of $2.3 \mathrm{~mm}$ at the midline to $3 \mathrm{~mm}$ at the periphery, ${ }^{8}$ again increasing the chances of trauma.

The safe donor area lies within and around the superior nuchal ridge where the occipital artery-nerve complex passes through. A lack of proper visualization and inadequate attention to the depth of incision while donor harvesting may result in injury to the occipital nerve and artery or any one of its branches. ${ }^{10,11}$

Complications such as bleeding, hematoma, the possibility of post surgical anagen effluvium, hypo-/hyperesthesia, neuromas, and neuralgias have been described. ${ }^{1,2}$

One of the most bothersome and irritating complications to these structures is occipital neuralgia. Treatment of this condition is difficult, ${ }^{6,11,13,14}$ therefore, the surgeon's knowledge of the anatomical course and landmarks for the identification of these structures is vital in preventing such complications.

There are several studies in the literature that address the location of the occipital artery-nerve complex. It has been shown that the nerve pierces the fascia of the trapezius muscle $37.8 \pm 4.6 \mathrm{~mm}$ from the occipital protuberance (range, $24-49 \mathrm{~mm}) .{ }^{5}$ In cadaveric studies, the distance between the midline and the point where the greater occipital nerve pierces the trapezius muscle aponeurosis (TMA) showed the right side having a mean of $35.4 \mathrm{~mm}$ and the left a mean of $33.9 \mathrm{~mm}$. A significant difference in the vertical distance from the occiput to the point at which the greater occipital nerve pierced the TMA was observed between male and female cadavers (male: mean, $11.4 \mathrm{~mm}$; range, 8.1-14.0; female: mean, $7.9 \mathrm{~mm}$; range, $3.9-11.3 \mathrm{~mm}$; SD, $1.9 \mathrm{~mm} ; \mathrm{p}=0.004) .^{4}$

These studies, however, mostly used bony landmarks as reference points.

Particular attention has been given to take into account existing anthropometric variations of the skull. ${ }^{8}$ In fact, reported failures of treating occipital neuralgia have been linked to these variations in skull anatomy. ${ }^{10}$ The occipital protuberance is a palpable bony landmark, not an exact spot, and this is even poorly established in some individuals. ${ }^{5}$

To eliminate this factor of skull variations, some studies have used two bony landmarks to locate the artery nerve complex. They have observed that the greater occipital nerve emerged from the aponeurosis of the trapezius muscle at $22 \%$ of the distance from the external occipital protuberance to the tip of the mastoid process or at $41 \%$ of the length of the intermastoid line. ${ }^{7}$ Locating the greater occipital nerve just medial to the palpated occipital artery and directing the injection needle $90^{\circ}$ towards the bony endpoint of the occiput for anaesthesia was proposed. ${ }^{7,13}$

In this study, we used the reflection of the skin of the external ear and scalp as our reference point because it is easily identifiable and more often than not present in all individuals. The maximal point of the occipital artery pulsations was usually felt at the level of the nuchal ridge corresponding to the area where the artery and nerve traverses the trapezius muscle aponeurosis. Pulsations can still be felt as the fingers are run superior to this point but are much weaker. This corresponds to the anatomic description of the ascending branches of the occipital artery where they can be found to run as high as the vertex of the scalp. ${ }^{10}$

In the general study population, the mean distance from the reflection of the skin of the external ear and scalp to the point of maximal occipital artery pulsations was $8.44 \mathrm{~cm}$ on the right and $8.42 \mathrm{~cm}$ on the left. The range from these mean measurements is $1.5-2 \mathrm{~cm}$ and indeed, upon donor harvesting in study patients, we did observe some arterial branches running right underneath the mark we had made or about $1-2 \mathrm{~cm}$ from the mark.

The Indian population showed the shortest distance at $7.18 \mathrm{~cm}$ on the right and $7.22 \mathrm{~cm}$ on the left. The farthest point of maximal occipital artery pulsations was recorded in Caucasians at $8.70 \mathrm{~cm}$ on the right and $8.61 \mathrm{~cm}$ on the left. The female population showed the shortest distance between occipital artery pulsations at $9.67 \mathrm{~cm}$. The longest distance between occipital artery pulsations was found in the patient of African descent at $11.50 \mathrm{~cm}$ followed by Caucasian patients at $11.04 \mathrm{~cm}$. It is important to keep in mind that only one patient of African descent was included in the study. The asymmetry in location of the right and left occipital artery pulsations was seen in $45 \%$ of the study population and was similar to studies showing $40 \%$ asymmetry between the right and left side as the occipital nerve exits the trapezius muscle aponeurosis. ${ }^{5}$

\section{Conclusion}

The findings reported in this study aim to enhance identification of the occipital nerve-artery complex using an easily identifiable reference point. Prior to donor harvesting and closure, it is advisable to always locate the occipital artery and nerve by the method of palpation used in this study. If pulsations are weak, it is difficult to palpate, or if some inconvenience is felt in finding its location, we recommend marking the area with a vertical line in the sagittal plane based on the mean measurements we report for the particular population. It is advised that within $1.5-2 \mathrm{~cm}$ from this point, dissection and undermining be very meticulously done since the occipital neurovascular bundle can be found to run underneath this vertical zone. We also recommend avoiding placement of deep or retention sutures within and surrounding this area. This can decrease chances of trauma to these structures and thus minimize complications.

It would be important to note and is likewise recommended that future studies include equal numbers of patients for each racial group, include measurements of total scalp circumference, and employ statistical analysis of data. It is hoped that the results from this study can offer a useful guide for hair transplant surgeons dealing with patients of multiracial groups to decrease chances of injury to the occipital artery and nerve complex during strip harvesting and donor closure.

\section{References}

1. Cooley, J. Complications of hair transplantation. In: W. Unger and R. Shapiro, eds. Hair Transplantation. New York: Marcel Dekker. 2004; 568-573. 
Multiracial variations

$\checkmark$ from page 129

2. Marzola, M., and Vogel, J. Complications. In: D. Stough and R. Haber, eds. Hair Transplantation. Philadelphia: Elsevier Saunders. 2006; 175-176.

3. Wolf, B. Anesthesia. In: W. Unger and R. Shapiro, eds. Hair Transplantation. New York: Marcel Dekker. 2004; 243.

4. Khan, S., and S. Khan. Nerve block and local anesthesia. In: D. Stough and R. Haber, eds. Hair Transplantation. Philadelphia: Elsevier Saunders. 2006; 80.

5. Ducic, I., M. Moriarty, and A. Al-Attar. Anatomical variations of the occipital nerves: implications for the treatment of chronic headaches. Plastic and Reconstruct Surg. 2009; 123:859-863.

6. Taylor, M., S. Silva, and C. Cottrell. Botulinum toxin-A in the treatment of occipital neuralgia: a pilot study. Headache. 2008; 48:1476-1481.

7. Loukas, M., et al. Identification of greater occipital nerve landmarks for the treatment of occipital neuralgia. Folia Morphol. 2006; 65:337-342.

8. Natsis, K., et al. The course of the greater occipital nerve in the suboccipital region. Clinical Anat. 2006; 19:332336.
9. Alvernia, J., et al. The occipital artery: a microanatomical study. Neurosurg. 2006; 58: 114-122.

10. Rose, P., R. Shapiro, and M. Morgan. Basic science. In: W. Unger and R. Shapiro, eds. Hair Transplantation, 4th ed. Chapter 2, p. 35.

11. Ducic, I., E. Hartmann, and E. Larson. Indications and outcomes for surgical treatment of patients with chronic migraine headaches caused by occipital neuralgia. Plastic and Reconstruct Surg. 2009; 123:1453-1461.

12. Shimizu, S., et al. Can proximity of the occipital artery to the greater occipital nerve act as a cause of idiopathic greater occipital neuralgia? An anatomical and histologic evaluation of the artery-nerve relationship. Plastic and Reconstruct Surg. 2007; 11 9:2029-2033.

13. Andrychowski, J., et al. Occipital neuralgia: possible failure of surgical treatment-case report. Folia Neuropathologica. 2009; 47:69-74.

14. Kapural, L., et al. Botulinum toxin occipital nerve block for the treatment of severe occipital neuralgia: a case series. Pain Practice. 2007; 7:337-340.

\section{Physicians \& Medical Assistants Wanted}

Hair Club, ${ }^{\circledR}$ the industry leader in hair restoration, is expanding and opening new centers throughout the USA.

We are seeking talented physicians and medical assistants with experience in follicular unit hair transplantation to work with us in our expanding markets.

If you are interested in working with a team that makes a difference in people's lives, you like to travel and enjoy working with motivated professionals, we invite you to contact us:

HR Department (800) 251-2658 Email: recruiting@hcfm.com www.hairclub.com

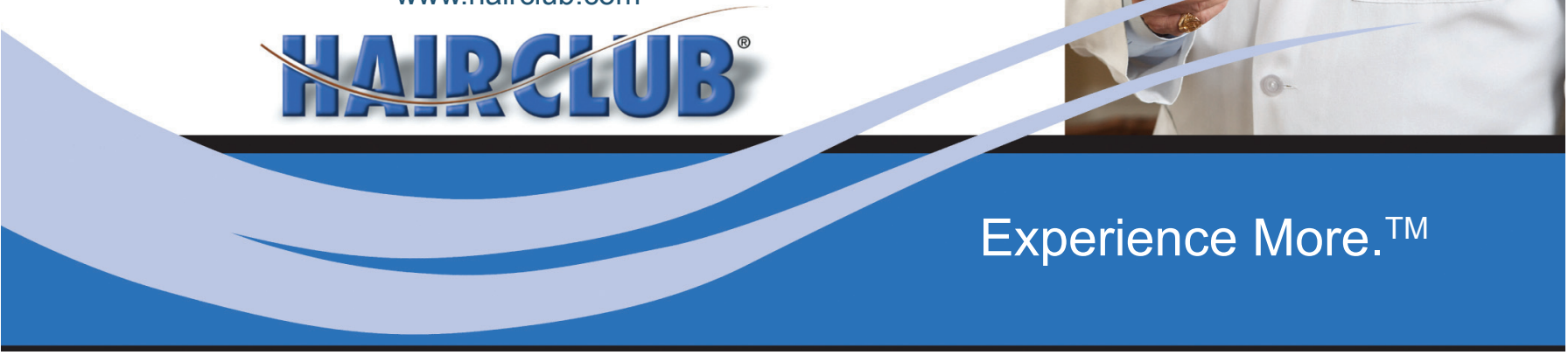

